\title{
Pancreatic metastasis from malignant phyllodes tumor of the breast
}

\author{
Seung Eun Lee ${ }^{1}$, Young Kyung Bae ${ }^{2}$, Joon Hyuk Choi ${ }^{2}$ \\ ${ }^{1}$ Department of Radiology, Yeungnam University College of Medicine, Daegu, Korea \\ ${ }^{2}$ Department of Pathology, Yeungnam University College of Medicine, Daegu, Korea
}

Received: September 7, 2020

Revised: October 30, 2020

Accepted: November 5, 2020

Corresponding author:

Seung Eun Lee

Department of Radiology,

Yeungnam University College of

Medicine, 170 Hyunchung-ro, Nam-

gu, Daegu 42415, Korea

Tel: +82-53-620-4129

Fax: +82-53-653-5484

E-mail: air1986@naver.com
Pancreatic metastasis from malignant phyllodes tumor (PT) of the breast is rare, and only a few cases have been reported in the literature. Here, we report a case of pancreatic metastasis from malignant PT of the breast in a 48-year-old woman. She had had three episodes of recurrence of malignant PT in her right breast. She presented with epigastric pain for 2 months. Computed tomography and magnetic resonance imaging revealed a $6 \mathrm{~cm}$-sized, well-defined, heterogeneous mass with peripheral enhancement in the body of the pancreas. Endoscopic ultrasonography-guided fine-needle aspiration was performed, and the pathologic report suggested spindle cell mesenchymal neoplasm. Subsequently, surgical excision was performed, and the mass was confirmed as a metastatic malignant PT. The imaging findings are discussed and the literature is briefly reviewed in this report.

Keywords: Breast; Neoplasm metastasis; Pancreas ; Phyllodes tumor

\section{Introduction}

Phyllodes tumors (PTs) are rare fibroepithelial neoplasms of the breast that account for $0.3 \%$ to $1 \%$ of all breast neoplasms [1]. PTs can be classified as benign, borderline, or malignant based on their histologic characteristics [2]. Malignant PTs have greater risk of distant metastasis than benign PTs. Distant metastases occur in $10 \%$ to $20 \%$ of patients with malignant PTs $[2,3]$. The lungs and bones are the most common sites of metastases via hematogenous spread $[1,4]$. The pancreas is an unusual site for metastasis, and only a few case reports were found in the literature [5-10]. Due to their rarity, the imaging findings of pancreatic metastases from PTs are extremely rare. Herein, we report a case of malignant PT that metastasized to the pancreas.

\section{Case}

This retrospective study was approved by the Institutional Review
Board (IRB) of the Yeungnam University Hospital (IRB No: 2020-05-053) and the requirement of informed consent from patient was waived by IRB.

A 48-year-old woman presented with epigastric pain for 2 months. The patient had previously suffered three episodes of recurrence of malignant PT in her right breast. She underwent right breast-conserving surgery with negative resection margins for malignant PT 7 years ago in 2013, and right nipple areolar skin sparing mastectomy for recurrent malignant PT in 2015. She subsequently underwent wide excision for recurrent malignant PT in 2018 (Fig. 1).

After 18 months from the last surgery, she was hospitalized with epigastric pain and abdominal computed tomography (CT) (Fig. 2) and magnetic resonance (MR) imaging (Fig. 3 ) showed a $6 \mathrm{~cm}$ sized heterogeneous enhancing mass involving the body of the pancreas. Her laboratory data, including the levels of the tumor markers carcinoembryonic antigen and carbohydrate antigen 19-9, were within normal range.

Copyright (C) 2021 Yeungnam University College of Medicine

This is an Open Access article distributed under the terms of the Creative Commons Attribution Non-Commercial License (http://creativecommons.org/licenses/by-nc/4.0/) which permits unrestricted non-commercial use, distribution, and reproduction in any medium, provided the original work is properly cited. 
Endoscopic ultrasonography-guided fine-needle aspiration was performed (Fig. 4), and the pathologic report suggested spindle cell mesenchymal neoplasm. Based on her history of malignant PT of her right breast, metastatic malignant PT was strongly suspected.

Open distal pancreatectomy with splenectomy was performed, and severe mesenteric adhesions surrounding the tumor were noted during the surgery. Histologic findings of the pancreatic tumor
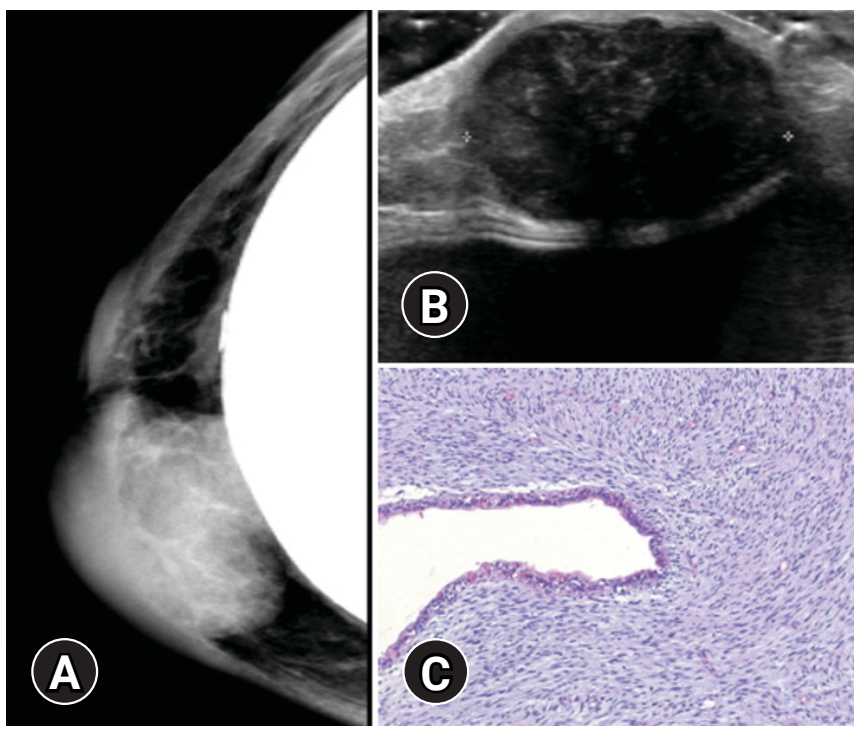

Fig. 1. Radiologic and pathologic findings of recurrent malignant phyllodes tumor of the breast in a 48-year-old woman. (A) Mammography of the right breast reveals an oval, circumscribed, hyperdense mass in the right inner breast, above the implant, with an approximate size of $3.6 \mathrm{~cm}$. (B) Ultrasonography reveals a $3.6 \times 3.0 \times 2.0 \mathrm{~cm}$-sized, oval, microlobulated, hypoechoic mass at the 3 o'clock location of the periareolar area. (C) The recurred breast mass shows stromal overgrowth and hypercellularity. The stromal cells are spindle-shaped and show significant nuclear atypia. Benign epithelial component is present (hematoxylin and eosin stain, $\times 100)$. were similar to those of the primary malignant PT of the breast (Fig. 5) and the tumor cells were positive for CD34. The final diagnosis was metastatic malignant PT. Following discussions with patient, she chose to proceed with adjuvant therapy with an immunomodulatory substance (Mesima; Han Kook Shin Yak, Nonsan, Korea), which enhances immunologic activities and anticancer effects. There was no evidence of recurrence during the first 4 months after surgery, but a long-term follow-up is now required.

\section{Discussion}

PTs are rare fibroepithelial neoplasms of the breast exhibit a wide range of clinical characteristics. The World Health Organization classifies PTs as benign, borderline, or malignant based on their histologic characteristics, including tumor margins, mitotic activity, degree of cellular atypia, stromal cellularity, and stromal overgrowth [2]. Histologically, PTs contain both epithelial and stromal components, and they have large leaf-like projections accompanied by high degree of increased stromal proliferation and cellularity. The stromal component has various histologic appearances that are correlated with metastasis and local recurrence [5]. In this case, the stromal overgrowth, high stromal cellularity, significant nuclear atypia of stromal cells, and the high mitotic count of 11 per 10 high-power fields could have led to distant metastasis.

Distant metastases occur in $10 \%$ to $20 \%$ of patients with malignant PTs. The most common sites of metastases are the lungs and bones $[2,3]$. However, several unusual metastatic sites, including duodenum, brain, nasal cavity, skin, oral cavity, skeletal muscle, mandible, and maxilla, have also been reported [11-13]. Metastasis to pancreas is rare, and only a few cases have been reported [5-10].

In the literature, the clinical symptoms of the previously reported cases of pancreatic metastases from malignant PTs, were obstructive jaundice, abdominal or back pain, hematemesis and intermittent tarry stools or steatorrhea. The mean age was 46 years
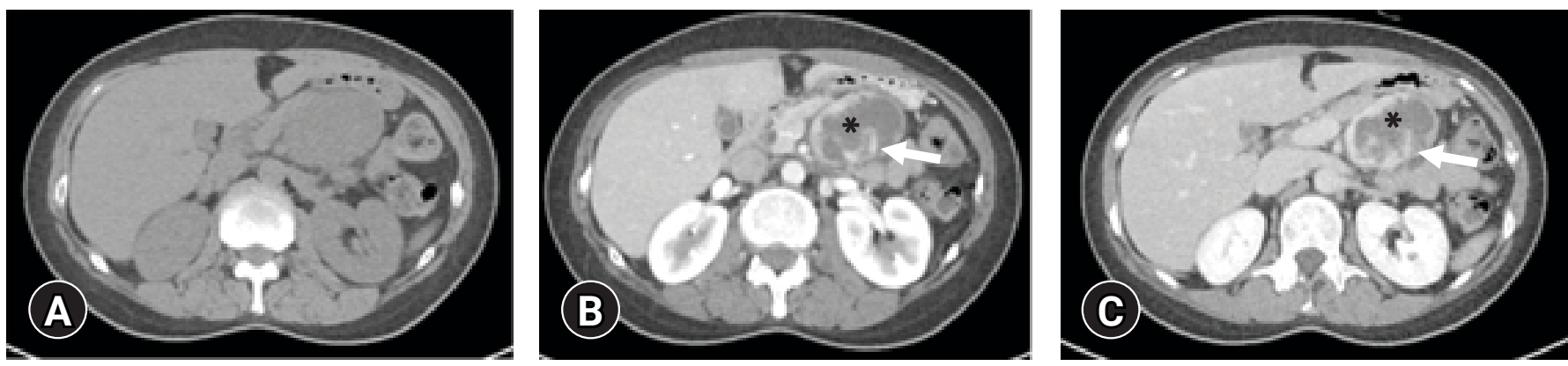

Fig. 2. Computed tomography imaging findings of pancreatic metastasis from malignant phyllodes tumor of the breast. Precontrast (A), arterial (B), and portal (C) phase of contrast-enhanced abdominal computed tomography show a $6 \mathrm{~cm}$-sized, well-defined heterogeneous mass involving the body of the pancreas. The mass reveals central cystic or necrotic portion (asterisk) and internal septa (arrow). The mass shows progressive, peripheral, and septal enhancement. 

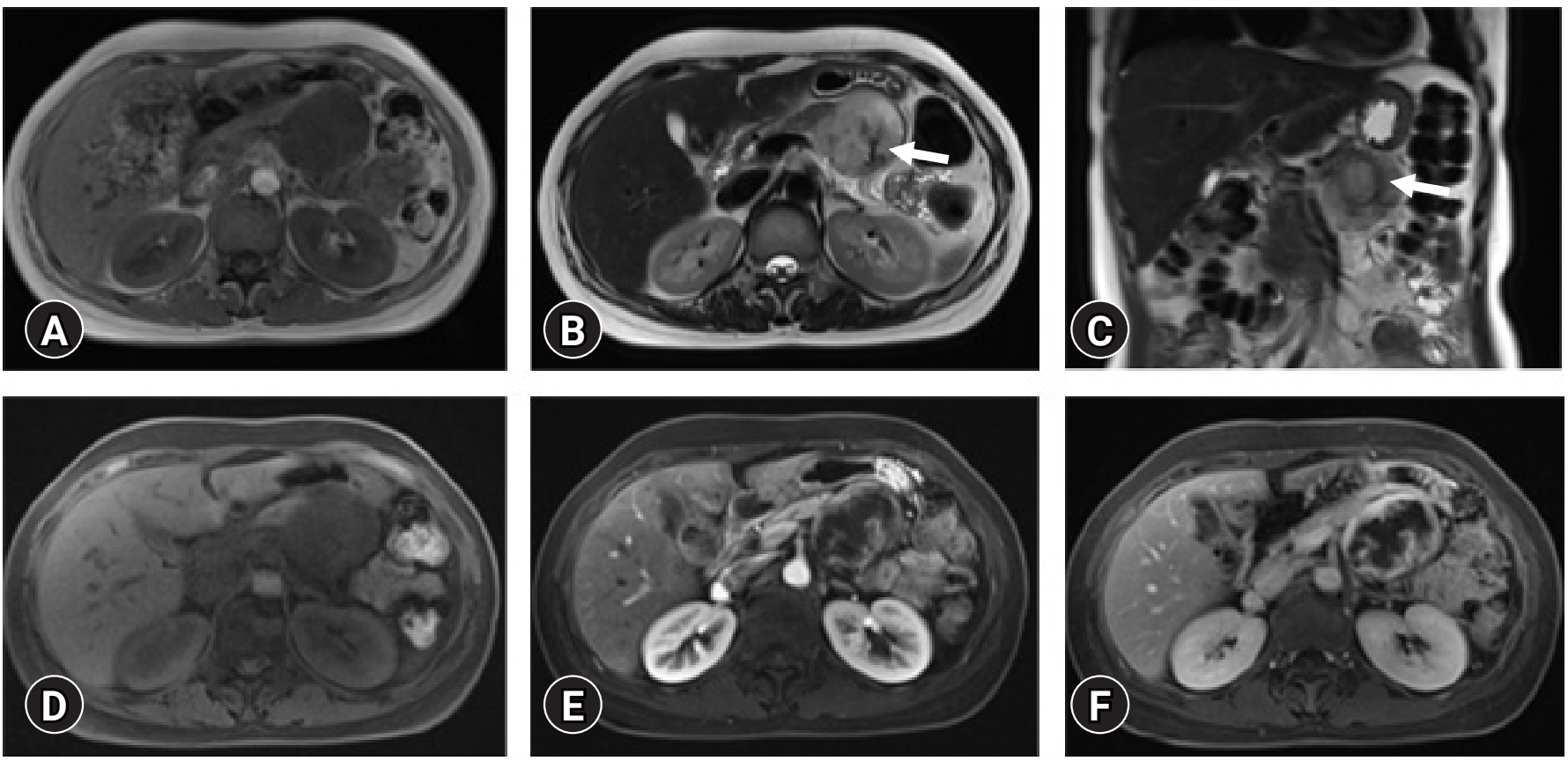

Fig. 3. Magnetic resonance imaging findings of pancreatic metastasis from malignant phyllodes tumor of the breast. The mass shows low signal intensity on T1-weighted image (A) and high signal intensity and internal septa with low signal intensities (arrows) on T2weighted images (B, C). (D-F) Dynamic studies after the administration of gadolinium show thick irregular peripheral enhancement and septal enhancement with central poorly enhancing areas.

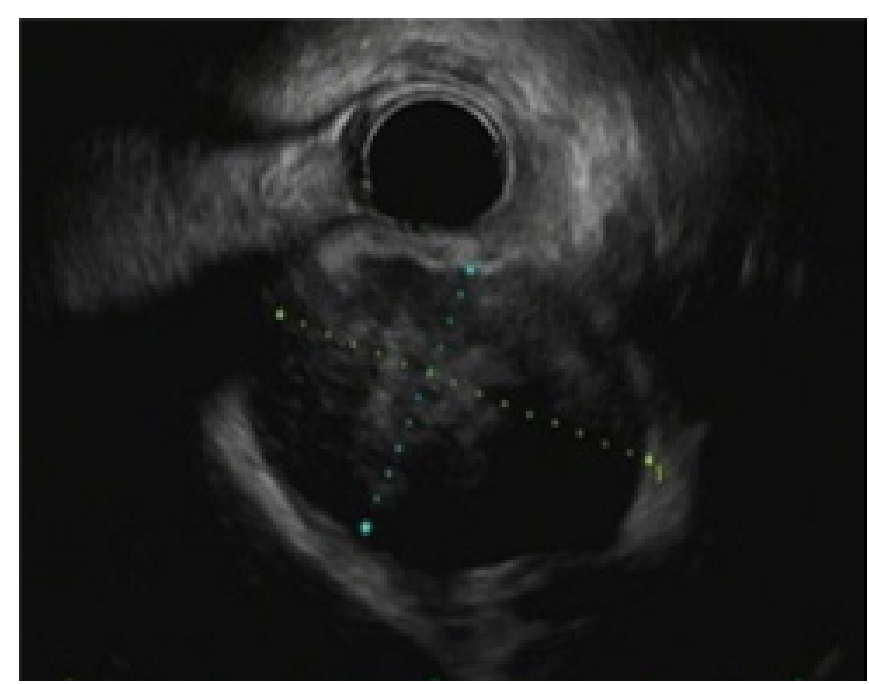

Fig. 4. Endoscopic ultrasonography (EUS) finding of pancreatic metastasis from malignant phyllodes tumor of the breast. EUS revealed a 6-cm mixed and heterogeneous hypoechoic mass in the body of the pancreas.

(range, 34-57 years) and the time to diagnosis from primary therapy was 3 years (range, 8 months- 6 years). In our case, the patient presented with epigastric pain for 2 months, and her age was within the range of previously reported cases. The time to diagnosis from primary therapy was approximately 6.5 years, which was the longest interval noted in the literature. However, our patient had had three episodes of recurrence, and metastasis was detected after 18 months from the last surgery. Therefore, the duration between diagnosis and the initial therapy was long, but the duration between diagnosis and the last surgery was comparable to what was reported for the previous cases.

Imaging findings were included in six case reports in the literature reviewed; six included CT images and only one included MR images (in Japanese) [5-10]. To our knowledge, this is the first report to discuss the MR imaging findings of pancreatic metastasis from PT in the English literature. Previous reports demonstrated hypodense mass (relatively to the pancreas) or peripheral enhancing mass with central low-density area on CT images [5-10]. There was a reported case accompanied by retroperitoneal hemorrhage on CT images [10]. The only MR images (in Japanese) presented in the available reports demonstrated a mass involving the pancreatic head and body with low signal intensity on T1-weighted image (T1WI) and high signal intensity on T2-weighted images. On MR cholangiopancreatography, the distal common bile duct was compressed by the mass, and the upstream biliary duct was dilated [9] In our case, the lesion appeared as an exophytic mass involving the pancreatic body without pancreatic or biliary duct dilatation. The mass revealed central cystic or necrotic portion and internal septa. The mass showed progressive peripheral and septal enhancement on dynamic studies of CT and MR imaging. The preoperative dif- 

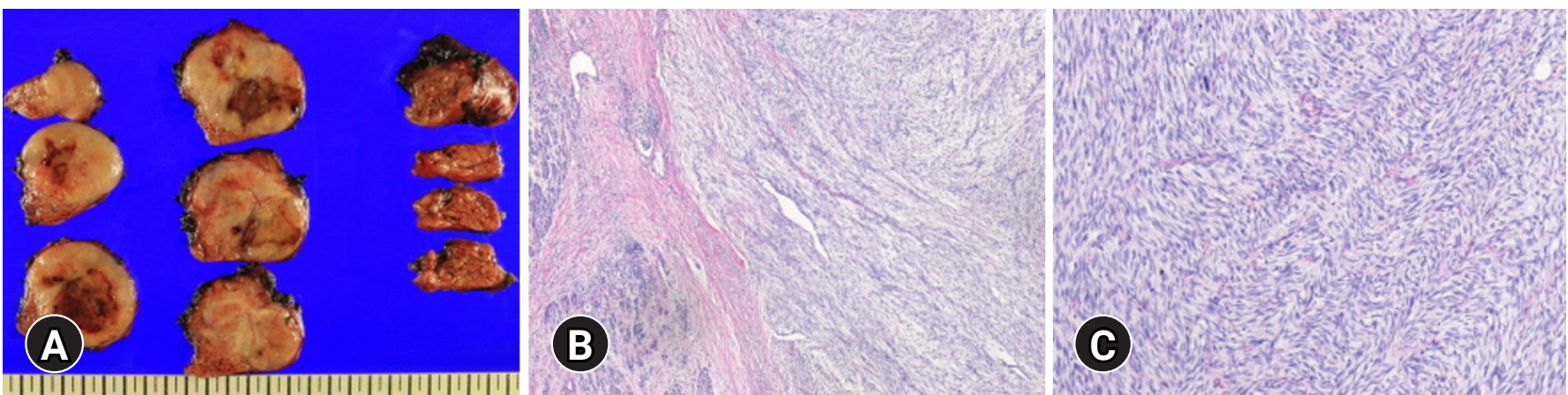

Fig. 5. (A) Gross photograph of the pancreatic tumor shows a 6.0×5.0-cm, grayish white, myxoid and solid cut surface with hemorrhage. (B) Histologic photographs of the pancreatic tumor show proliferation of spindle-shaped tumor cells in the myxoid stroma. (C) The tumor cells have elongated hyperchromatic nuclei, with fascicular pattern (hematoxylin and eosin stain, x100 [B] and x200 [C]).

ferential diagnoses included pancreatic metastasis, solid pseudopapillary neoplasm, and neuroendocrine tumor with cystic degeneration. Solid pseudopapillary neoplasms are often difficult to differentiate from other solid tumors of the pancreas when they are small, and the cystic changes are insignificant. Solid pseudopapillary neoplasms usually contain hemorrhage that may have high signal intensity on T1WI; however, the mass showed homogeneous low signal intensity on T1WI in this case. Neuroendocrine tumors show more rapid enhancement during the arterial phase due to an abundant vascular supply, but in our case, there was progressive enhancement on dynamic studies [14]. The radiologic findings of pancreatic metastases are usually similar to those of primary carcinomas, but they are nonspecific, so the past history of malignancy is helpful for diagnosis.

The standard treatment for distant metastases from PTs has not been established yet, because of lack of large prospective studies. Due to the low incidence of metastatic PTs, only a small number of retrospectively analyzed cases have been reported. Furthermore, treatment options, including surgery, radiotherapy, hormone therapy, and chemotherapy, remain limited. In previous case reports, patients underwent chemotherapy with a diverse regimen [5-10]. Bednar et al. [8] reported that patients with isolated pancreatic metastasis from breast cancer who undergo resection have the potential for prolonged postresection survival. They suggested that definite curative pancreatic resection can be a viable treatment option for patients with isolated metastasis to the pancreas. In our case, the patient had metastasis only to the pancreas, and she underwent surgical resection. The prognosis of patients with distant metastases from PTs is poor, with an average survival time of less than 2 years $[2,15]$.

In summary, we report a case of pancreatic metastasis from malignant PT of the breast. Although it is rare, metastatic PT should be considered in differential diagnosis when evaluating a pancreat- ic mass in the patient with history of malignant PT of the breast.

\section{Acknowledgments}

\section{Conflicts of interest}

No potential conflict of interest relevant to this article was reported.

\section{Author contributions}

Conceptualization, Project administration: SEL; Data curation: all authors; Writing-original draft: SEL, JHC; Writing-review \& editing: all authors.

\section{ORCID}

Seung Eun Lee, https://orcid.org/0000-0001-6693-4752

Young Kyung Bae, https://orcid.org/0000-0002-6689-9413

Joon Hyuk Choi, https://orcid.org/0000-0002-8638-0360

\section{References}

1. Telli ML, Horst KC, Guardino AE, Dirbas FM, Carlson RW. Phyllodes tumors of the breast: natural history, diagnosis, and treatment. J Natl Compr Canc Netw 2007;5:324-30.

2. Mishra SP, Tiwary SK, Mishra M, Khanna AK. Phyllodes tumor of breast: a review article. ISRN Surg 2013;2013:361469.

3. Zhou ZR, Wang CC, Yang ZZ, Yu XL, Guo XM. Phyllodes tumors of the breast: diagnosis, treatment and prognostic factors related to recurrence. J Thorac Dis 2016;8:3361-8.

4. Kessinger A, Foley JF, Lemon HM, Miller DM. Metastatic cystosarcoma phyllodes: a case report and review of the literature. J Surg Oncol 1972;4:131-47.

5. Amir RA, Rabah RS, Sheikh SS. Malignant phyllodes tumor of the breast with metastasis to the pancreas: a case report and re- 
view of literature. Case Rep Oncol Med 2018;2018:6491675.

6. Ang TL, Ng VW, Fock KM, Teo EK, Chong CK. Diagnosis of a metastatic phyllodes tumor of the pancreas using EUS-FNA. JOP 2007;8:35-8.

7. Bachert SE, Stewart RL, Samayoa L, Massarweh SA. Malignant phyllodes tumor metastatic to pancreas. Breast J 2020;26: 1627-8.

8. Bednar F, Scheiman JM, McKenna BJ, Simeone DM. Breast cancer metastases to the pancreas. J Gastrointest Surg 2013; 17:1826-31.

9. Serikawa M, Sasaki T, Kobayashi K, Itsuki H, Kamigaki M, Minami T, et al. Malignant phyllodes tumor metastatic to the pancreas: a case report. Nihon Shokakibyo Gakkai Zasshi 2012; 109:795-803.

10. Yukawa M, Watatani M, Isono S, Shiono H, Hasegawa H, Okajima K, et al. Pancreatic metastasis from phyllodes tumor presenting initially as acute retroperitoneal hemorrhage. Int Canc Conf J 2013;2:238-42.
11. Asoglu O, Karanlik H, Barbaros U, Yanar H, Kapran Y, Kecer M, et al. Malignant phyllode tumor metastatic to the duodenum. World J Gastroenterol 2006; 12:1649-51.

12. Karczmarek-Borowska B, Bukala A, Syrek-Kaplita K, Ksiazek M, Filipowska J, Gradalska-Lampart M. A rare case of breast malignant phyllodes tumor with metastases to the kidney: case report. Medicine (Baltimore) 2015;94:e1312.

13. Khangembam BC, Sharma P, Singla S, Singhal A, Dhull VS, Bal $\mathrm{C}$, et al. Malignant phyllodes tumor of the breast metastasizing to the vulva: (18)F-FDG PET-CT demonstrating rare metastasis from a rare tumor. Nucl Med Mol Imaging 2012;46:232-3.

14. Low G, Panu A, Millo N, Leen E. Multimodality imaging of neoplastic and nonneoplastic solid lesions of the pancreas. Radiographics 2011;31:993-1015.

15. Mituś JW, Blecharz P, Walasek T, Reinfuss M, Jakubowicz J, Kulpa J. Treatment of patients with distant metastases from phyllodes tumor of the breast. World J Surg 2016;40:323-8. 